\title{
Resonance capture and dynamics of three-planet systems
}

\author{
C. Charalambous, ${ }^{1 \star}$ J. G. Martí, ${ }^{2}$ C. Beaugé ${ }^{1 \star}$ and X. S. Ramos ${ }^{1}$ \\ ${ }^{1}$ Instituto de Astronomía Teórica y Experimental, Observatorio Astronómico, Universidad Nacional de Córdoba, Laprida 854, Córdoba X5000GBR, \\ Argentina \\ ${ }^{2}$ Instituto de Astrofísica de La Plata, Facultad de Ciencias Astronómicas y Geofísicas, Universidad Nacional de La Plata, La Plata B1900FWA, Argentina
}

Accepted 2018 March 6. Received 2018 March 6; in original form 2017 December 22

\begin{abstract}
We present a series of dynamical maps for fictitious three-planet systems in initially circular coplanar orbits. These maps have unveiled a rich resonant structure involving two or three planets, as well as indicating possible migration routes from secular to double resonances or pure three-planet commensurabilities. These structures are then compared to the present-day orbital architecture of observed resonant chains. In a second part of the paper, we describe $N$-body simulations of type-I migration. Depending on the orbital decay time-scale, we show that three-planet systems may be trapped in different combinations of independent commensurabilities: (i) double resonances, (ii) intersection between a two-planet and a first-order three-planet resonances, and (iii) simultaneous libration in two first-order three-planet resonances. These latter outcomes are found for slow migrations, while double resonances are almost always the final outcome in high-density discs. Finally, we discuss an application to the TRAPPIST-1 system. We find that, for low migration rates and planetary masses of the order of the estimated values, most three-planet sub-systems are able to reach the observed double resonances after following evolutionary routes defined by pure three-planet resonances. The final orbital configuration shows resonance offsets comparable with present-day values without the need of tidal dissipation. For the $8 / 5$ resonance proposed to dominate the dynamics of the two inner planets, we find little evidence of its dynamical significance; instead, we propose that this relation between mean motions could be a consequence of the interaction between a pure three-planet resonance and a two-planet commensurability between planets $\mathrm{c}$ and $\mathrm{d}$.
\end{abstract}

Key words: methods: numerical - celestial mechanics - planets and satellites: dynamical evolution and stability.

\section{INTRODUCTION}

TRAPPIST-1 (Gillon et al. 2016, 2017; Luger et al. 2017) is a unique exoplanetary system of seven planets in a complex resonant chain comprised of five interlocked zero-order (i.e. Laplace) threebody mean-motion resonances (MMRs). Although the multiresonant state is not yet confirmed and most initial conditions consistent with the observations lead to dynamical instabilities in short timescales (Gillon et al. 2017), $N$-body simulations by Tamayo et al. (2017) indicated that similar stable configurations may be reached by smooth planetary migration.

In recent years, several transit systems have been discovered in multiplanet resonances: Kepler-60 (Steffen et al. 2013; Goździewski et al. 2016), Kepler-80 (MacDonald et al. 2016), and most noticeably Kepler-223 (Mills et al. 2016) where precise TTVs spanning over $4 \mathrm{yr}$ of observations has shown the actual libration

^E-mail: charalambous@oac.unc.edu.ar (CC); beauge@oac.unc.edu.ar (CB) of the Laplace angles. Independently of the known number of planets, the fundamental building blocks of all these resonance chains consist of three-body Laplace resonances. Thus, independent of the planet multiplicity, many dynamical properties of multiresonant systems may be tackled by studying three-planet commensurabilities.

A particularly interesting case is Kepler-444 (Campante et al. 2015), with five planets orbiting the host star within 0.8 au. This is a noteworthy system for two reasons. First, the central star has a stellar companion at $\sim 60$ au, making the binary sufficiently tight to have influenced the dynamical evolution and, possibly, the formation process itself. Second, the age of host star is estimated at 11.2 Gyr, one of the oldest planetary systems known to date, and thus, a good candidate to analyse how tidal effects may have altered its primordial orbital architecture.

Three-planet resonances may also play a relevant role in defining the orbital architecture of our own Solar system. Apart from the classical Laplace resonance between the inner three Galilean satellites (e.g. Yoder 1979), resonant chains have also been proposed as acting between the outer planets (Murray \& Holman 1999; Guzzo 
2005, 2006) possibly leading to (extremely weak) chaotic motion in the outer Solar system.

In this work, we will present a series of dynamical maps of the orbital-period ratio representative plane of initial conditions for three-planet systems. These will help unveil the complex richness of resonant structures as well as the relative strengths and common origin between two-planet and several different types of three-planet resonances. In a second part, we study the migration and resonance capture of the TRAPPIST- 1 system and analyse outcomes for fictitious systems as a function of the initial conditions, planetary masses, and migration rates. Finally, a discussion is presented in how different types of resonant configurations may be reached in each case.

\section{THE DYNAMICAL SYSTEM}

\subsection{Variables}

Our dynamical system consists of three planetary masses $m_{\mathrm{i}}(i=1$, $2,3)$ in coplanar orbits around a central star of mass $m_{0}$, with $m_{0}>m_{\mathrm{i}}$. We will denote with $a_{\mathrm{i}}$ the semimajor axes, $e_{\mathrm{i}}$ the eccentricities, $\lambda_{i}$ the mean longitudes, and $\varpi_{i}$ the longitudes of pericentre of each planet. All orbital elements are defined in a Jacobi reference frame.

Since our analytical model will be based on a Hamiltonian formalism, it is useful to first introduce the modified Delaunay canonical variables which, in the planar problem, are given by:

$L_{\mathrm{i}}=m_{\mathrm{i}}^{\prime} \sqrt{\mu_{\mathrm{i}} a_{\mathrm{i}}} ; \lambda_{\mathrm{i}}$

$S_{\mathrm{i}}=L_{\mathrm{i}}\left(1-\sqrt{1-e_{\mathrm{i}}^{2}}\right) ;-\varpi_{\mathrm{i}}$

where the mass factors acquire the form:

$\mu_{\mathrm{i}}=\mathcal{G} \sum_{j=0}^{i} m_{\mathrm{j}} ; m_{\mathrm{i}}^{\prime}=m_{\mathrm{i}} \frac{\sum_{j=0}^{i-1} m_{\mathrm{j}}}{\sum_{j=0}^{i} m_{\mathrm{j}}}$,

the latter being the reduced mass of the $i$ th planet. The gravitational constant is denoted by $\mathcal{G}$.

The Hamiltonian $F$ for the system can then be written as the sum of two terms $F=F_{0}+F_{1}$; the first leads to the Keplerian motion of the planets around the central star, while $F_{1}$ groups all perturbations arising from mutual gravitational interactions between the planets. Written in the Delaunay variables (1), the integrable Hamiltonian $F_{0}$ acquires de form:

$F_{0}=-\sum_{i=1}^{N} \frac{\mu_{\mathrm{i}}^{2} m_{\mathrm{i}}^{\prime 3}}{2 L_{\mathrm{i}}^{2}}=-\frac{\mu_{1}^{2} m_{1}^{\prime 3}}{2 L_{1}^{2}}-\frac{\mu_{2}^{2} m_{2}^{\prime 3}}{2 L_{2}^{2}}-\frac{\mu_{3}^{2} m_{3}^{\prime 3}}{2 L_{3}^{2}}$,

while the perturbation term can be generically expressed as:

$F_{1} \equiv-\mathcal{R}=-\mathcal{R}_{12}-\mathcal{R}_{23}-\mathcal{R}_{13}$,

where $\mathcal{R}_{\mathrm{ij}}$ denotes the disturbing function that arises from the interaction between $m_{\mathrm{i}}$ and $m_{\mathrm{j}}$. Retaining only terms corresponding to the lowest order of the masses, the gravitational perturbations have the same functional form as the one deduced for the restricted three-body problem (e.g. Libert \& Henrard 2007). Thus, the planetary disturbing function may be expressed in terms of the position vectors $\boldsymbol{r}_{\mathrm{i}}$ as:

$\mathcal{R}_{\mathrm{ij}}=\mathcal{G} m_{\mathrm{i}} m_{\mathrm{j}}\left(\frac{1}{\left|\boldsymbol{r}_{\mathrm{i}}-\boldsymbol{r}_{\mathrm{j}}\right|}-\frac{\boldsymbol{r}_{\mathrm{i}} \cdot \boldsymbol{r}_{\mathrm{j}}}{\left|\boldsymbol{r}_{\mathrm{j}}\right|^{3}}\right)$,

where $\boldsymbol{r}_{\mathrm{i}}$ are in the Jacobi reference frame.

\subsection{Transformation between mean and osculating elements}

All resonant conditions are defined in mean variables (i.e. averaged over short-period terms), but our dynamical maps will be calculated in a representative plane of osculating elements. While the difference between both sets may not be significant for low planetary masses and/or for systems far from the Hill stability limit (e.g. Ferraz-Mello, Michtchenko \& Beaugé 2005; Deck, Payne \& Holman 2013; Ramos, Correa-Otto \& Beaugé 2015), it will prove important to correctly identify the resonances appearing in the dynamical maps and to estimate their relative strength.

Although the details of the canonical transformation for twoplanet systems are well documented (e.g. Tisserand 1889; Deck et al. 2013; Ramos et al. 2015), the extension to three-planet systems are not easily available and will be given here. The steps for the construction of the generating function are analogous, the only significant difference is the existence of three independent terms in the disturbing function (5). We follow, thus, the procedure employed by Ramos et al. (2015) extended to the case of three planets.

Let us denote by $\left(L_{\mathrm{i}}, S_{\mathrm{i}}, \lambda_{\mathrm{i}},-\varpi_{\mathrm{i}}\right)$ the osculating set of variables, while the mean canonical elements will be expressed by $\left(L_{\mathrm{i}}^{*}, S_{\mathrm{i}}^{*}, \lambda_{\mathrm{i}}^{*},-\varpi_{\mathrm{i}}^{*}\right)$. We then search for a Lie-type generating function $B:\left(L_{\mathrm{i}}, S_{\mathrm{i}}, \lambda_{\mathrm{i}},-\varpi_{\mathrm{i}}\right) \rightarrow\left(L_{\mathrm{i}}^{*}, S_{\mathrm{i}}^{*}, \lambda_{\mathrm{i}}^{*},-\varpi_{\mathrm{i}}^{*}\right)$ such that the transformed Hamiltonian is independent of the new mean longitudes, i.e. $F^{\star}=F^{\star}\left(S_{\mathrm{i}}^{*},-\varpi_{\mathrm{i}}^{*} ; L_{\mathrm{i}}^{*}\right)$. Up to lowest order in the masses, the relation between both sets of variables will be explicitly given by:

$L_{\mathrm{i}}=L_{\mathrm{i}}^{*}+\frac{\partial B}{\partial \lambda_{\mathrm{i}}} ; \lambda_{\mathrm{i}}=\lambda_{\mathrm{i}}^{*}-\frac{\partial B}{\partial L_{\mathrm{i}}}$

$S_{\mathrm{i}}=S_{\mathrm{i}}^{*}-\frac{\partial B}{\partial \varpi_{\mathrm{i}}} ; \varpi_{\mathrm{i}}=\varpi_{\mathrm{i}}^{*}+\frac{\partial B}{\partial S_{\mathrm{i}}}$,

with $(i=1,2,3)$. The first-order generating function $B$ is the solution of the partial differential equation

$-\boldsymbol{n} \cdot \frac{\partial B}{\partial \lambda}=\left\{F_{1}\right\}=-\left\{\mathcal{R}_{12}\right\}-\left\{\mathcal{R}_{23}\right\}-\left\{\mathcal{R}_{13}\right\}$

where $\boldsymbol{n}=\left(n_{1}, n_{2}, n_{3}\right)$ is the mean-motion vector, $\boldsymbol{\lambda}=\left(\lambda_{1}, \lambda_{2}, \lambda_{3}\right)$ is the mean longitudes vector, and $\left\{\mathcal{R}_{\mathrm{ij}}\right\}$ denotes the purely periodic part of $\mathcal{R}_{\mathrm{ij}}$ (e.g. Hori 1961; Ferraz-Mello 2007). Since we have chosen to express $B$ in osculating variables, the transformation equations (6) will have to be solved iteratively; however, the precision gained by this approach makes the extra work worthwhile.

In case of circular orbits, and neglecting the indirect terms, the Laplace expansion of the disturbing function acquires the form (e.g. Brouwer \& Clemence 1961)

$\mathcal{R}_{\mathrm{ij}}=\frac{\mathcal{G} m_{\mathrm{i}} m_{\mathrm{j}}}{2 a_{\mathrm{j}}} \sum_{k=-\infty}^{\infty} b_{1 / 2}^{(k)}\left(\alpha_{\mathrm{ij}}\right) \cos k\left(\lambda_{\mathrm{i}}-\lambda_{\mathrm{j}}\right)$

where $\alpha_{\mathrm{ij}}=a_{\mathrm{i}} / a_{\mathrm{j}}$ are the semimajor-axes ratios. Adding up the three different gravitational functions, eliminating the secular (i.e. non-periodic) terms, and introducing the resulting expression into equation (7), we can explicitly calculate the generating function, yielding

$$
\begin{aligned}
B= & \frac{\mathcal{G} m_{1} m_{2}}{a_{2}\left(n_{1}-n_{2}\right)} \sum_{k=1}^{\infty} \frac{1}{k} b_{1 / 2}^{(k)}\left(\alpha_{12}\right) \sin k\left(\lambda_{1}-\lambda_{2}\right) \\
& +\frac{\mathcal{G} m_{2} m_{3}}{a_{3}\left(n_{2}-n_{3}\right)} \sum_{k=1}^{\infty} \frac{1}{k} b_{1 / 2}^{(k)}\left(\alpha_{23}\right) \sin k\left(\lambda_{2}-\lambda_{3}\right) \\
& +\frac{\mathcal{G} m_{1} m_{3}}{a_{3}\left(n_{1}-n_{3}\right)} \sum_{k=1}^{\infty} \frac{1}{k} b_{1 / 2}^{(k)}\left(\alpha_{13}\right) \sin k\left(\lambda_{1}-\lambda_{3}\right) .
\end{aligned}
$$


Since we have adopted circular orbits, $B$ does not depend on either $S$ or $\varpi$, leading to identical values in both sets of variables. Moreover, choosing initial conditions with $\lambda_{\mathrm{i}}=0$ also leads to $\lambda_{\mathrm{i}}^{*}=0$, while the change in the Delaunay action $L_{\mathrm{i}}$ may be written in terms of the original form of the disturbing functions as:

$L_{1}=L_{1}^{*}+\frac{\left\{\mathcal{R}_{12}\right\}}{\left(n_{1}-n_{2}\right)}+\frac{\left\{\mathcal{R}_{13}\right\}}{\left(n_{1}-n_{3}\right)}$

$L_{2}=L_{2}^{*}-\frac{\left\{\mathcal{R}_{12}\right\}}{\left(n_{1}-n_{2}\right)}+\frac{\left\{\mathcal{R}_{23}\right\}}{\left(n_{2}-n_{3}\right)}$

$L_{3}=L_{3}^{*}-\frac{\left\{\mathcal{R}_{13}\right\}}{\left(n_{1}-n_{3}\right)}-\frac{\left\{\mathcal{R}_{23}\right\}}{\left(n_{2}-n_{3}\right)}$.

Since $\lambda_{\mathrm{i}}=0$, the amplitude of the periodic functions can be written explicitly as

$$
\begin{aligned}
\left\{\mathcal{R}_{\mathrm{ij}}\right\} & =\frac{\mathcal{G} m_{\mathrm{i}} m_{\mathrm{j}}}{a_{\mathrm{j}}}\left(\frac{a_{\mathrm{j}}}{\Delta_{\mathrm{ij}}}-\frac{1}{2} b_{1 / 2}^{(0)}\left(\alpha_{\mathrm{ij}}\right)\right) \\
= & \frac{\mathcal{G} m_{\mathrm{i}} m_{\mathrm{j}}}{a_{\mathrm{j}}}\left(\frac{1}{\left(1-\alpha_{\mathrm{ij}}\right)}-\frac{1}{2} b_{1 / 2}^{(0)}\left(\alpha_{\mathrm{ij}}\right)\right),
\end{aligned}
$$

where $\Delta_{\mathrm{ij}}=\left|\boldsymbol{r}_{\mathrm{i}}-\boldsymbol{r}_{\mathrm{j}}\right|$. Introducing this expression into equation (10), we can obtain closed analytical formula for the transformation between mean and osculating Delaunay momenta and, consequently, between the semimajor axes.

However, an additional simplification may be performed. Inspired by the surprising linear correlation found by Wisdom (1980) between the amplitude of the main resonant term and the degree $p$ of a given first-order resonance, we searched for a similar trend for the short-period terms. Although we failed to find a similar expression for the Laplace coefficient itself, we did find a suitable approximation for the full amplitude of the short-period perturbation equation (11). Explicitly we found that

$$
\frac{1}{1-\alpha_{\mathrm{ij}}}-\frac{1}{2} b_{1 / 2}^{0}\left(\alpha_{\mathrm{ij}}\right) \simeq 1.43 p_{\mathrm{ij}}+0.13 \text {, }
$$

where the numerical coefficients were determined using a leastsquares linear fit in the parameter $p_{\mathrm{ij}}$, defined as

$$
p_{\mathrm{ij}}=\frac{\alpha_{\mathrm{ij}}^{3 / 2}}{1-\alpha_{\mathrm{ij}}^{3 / 2}} \text {. }
$$

When initial conditions place the semimajor axis ratio in a firstorder resonance, then $p_{\mathrm{ij}}$ is an integer and equal to the degree of that commensurability, i.e. $n_{\mathrm{i}} / n_{\mathrm{j}}=\left(p_{\mathrm{ij}}+1\right) / p_{\mathrm{ij}}$. In any other configuration $p_{\mathrm{ij}}$ takes non-integer values. Fig. 1 compares the predictions of equation (12) (dashed curve) with the exact values (broad grey line), while the inlaid plot highlights the relative error $\epsilon$ between both. The linear fit guarantees a maximum relative error $\epsilon \sim 10^{-2}$ for all orbital separations in the interval $\alpha \in[0.3,0.9]$.

\section{RESONANT STRUCTURE}

\subsection{Dynamical maps}

We begin with a numerical study of the resonant structure of the three-planet problem. This will be accomplished by means of a series of dynamical maps in the $\left(n_{1} / n_{2}, n_{2} / n_{3}\right)$ plane, with initial conditions corresponding to circular planar orbits with all angles equal to zero. This choice corresponds to a collinear configuration where the mutual distance between the planets is minimum.

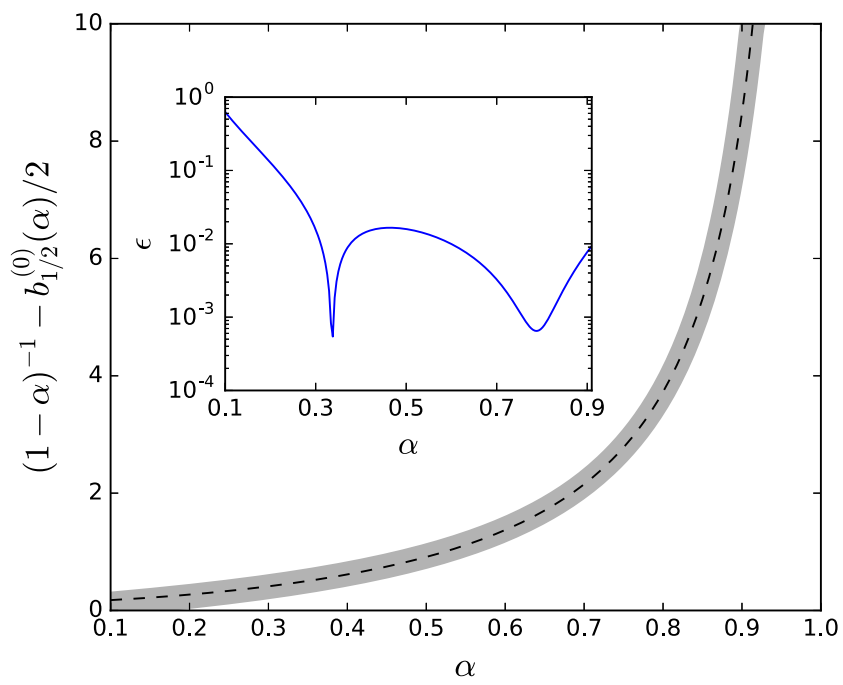

Figure 1. Broad grey curve in the main plot shows the function $(1-\alpha)^{-1}-$ $(1 / 2) b_{1 / 2}^{(0)}(\alpha)$ as function of the semimajor axis ratio, while the thin dashed curve corresponds to the approximation described in equation (12). The inlaid graph plots the relative error $\epsilon$ of the minimum-squares fit.

In our numerical simulations, we integrated the equations of motions of the four bodies of the system (central mass plus three planets) in a Jacobi reference frame, using a Bulrisch-Stöer algorithm with a precision specified by a maximum permitted relative error per time-step of $10^{-13}$. We set the central mass to $m_{0}=1 \mathrm{~m}_{\odot}$ and chose the initial $a_{3}$ to be always equal to $1 \mathrm{au}$. Each initial configuration with different initial semimajor axis ratios was integrated for a total time span of $T=10^{4} \mathrm{yr}$ (which in this case represent the total orbits of the outer body). During the integrations we kept track of the variation of each planet's semimajor axis, being able to calculate at the end each planet's maximum variation during the whole timespan, $\Delta a_{\mathrm{i}}=\left(a_{\mathrm{imax}}-a_{\mathrm{imin}}\right)$ (e.g. Gallardo, Coito \& Badano 2016). We also calculated for each initial condition the maximum value of $\Delta a$, which is the maximum of the planetary variations: $\max (\Delta a)=\max \left(\Delta a_{1}, \Delta a_{2}, \Delta a_{3}\right)$. Although this indicator does not measure chaotic motion, it is useful for mapping the resonant structure and analysing the behaviour of planetary systems, very similar to the better known maximum eccentricity method (e.g. Dvorak et al. 2004; Ramos et al. 2015). The $\max (\Delta a)$ measure was chosen over its $\max (\Delta e)$ counterpart, since it better identifies Laplace-type resonances, where the eccentricity suffers no appreciable excitation.

The top frame of Fig. 2 shows a dynamical map calculated over a grid of $800 \times 800$ initial conditions where all three masses were taken equal to $m_{\mathrm{i}}=150 m_{\oplus}$. The colour code indicates the value of $\max (\Delta a)$ after $T=10^{4} \mathrm{yr}$. Blue corresponds to small changes in the semimajor axes (usually indicative of regular motion), while red indicates large variations. These may correspond either to dynamically unstable orbits (escapes or collisions) or to stable initial conditions close to resonant separatrix, whose dynamics led to high eccentricities. The integration time was chosen sufficiently large to map the main features of the resonant structure but not so long so as to blur them with chaotic diffusion. Thus, at this point, we are more interested in mapping the phase space than in identifying stable/unstable domains.

The phase plane shows a rich structure generated by a web of two- and three-planet resonances. All commensurabilities in this plane are characterized by a condition of type

$j_{1} n_{1}+j_{2} n_{2}+j_{3} n_{3} \simeq 0$, 

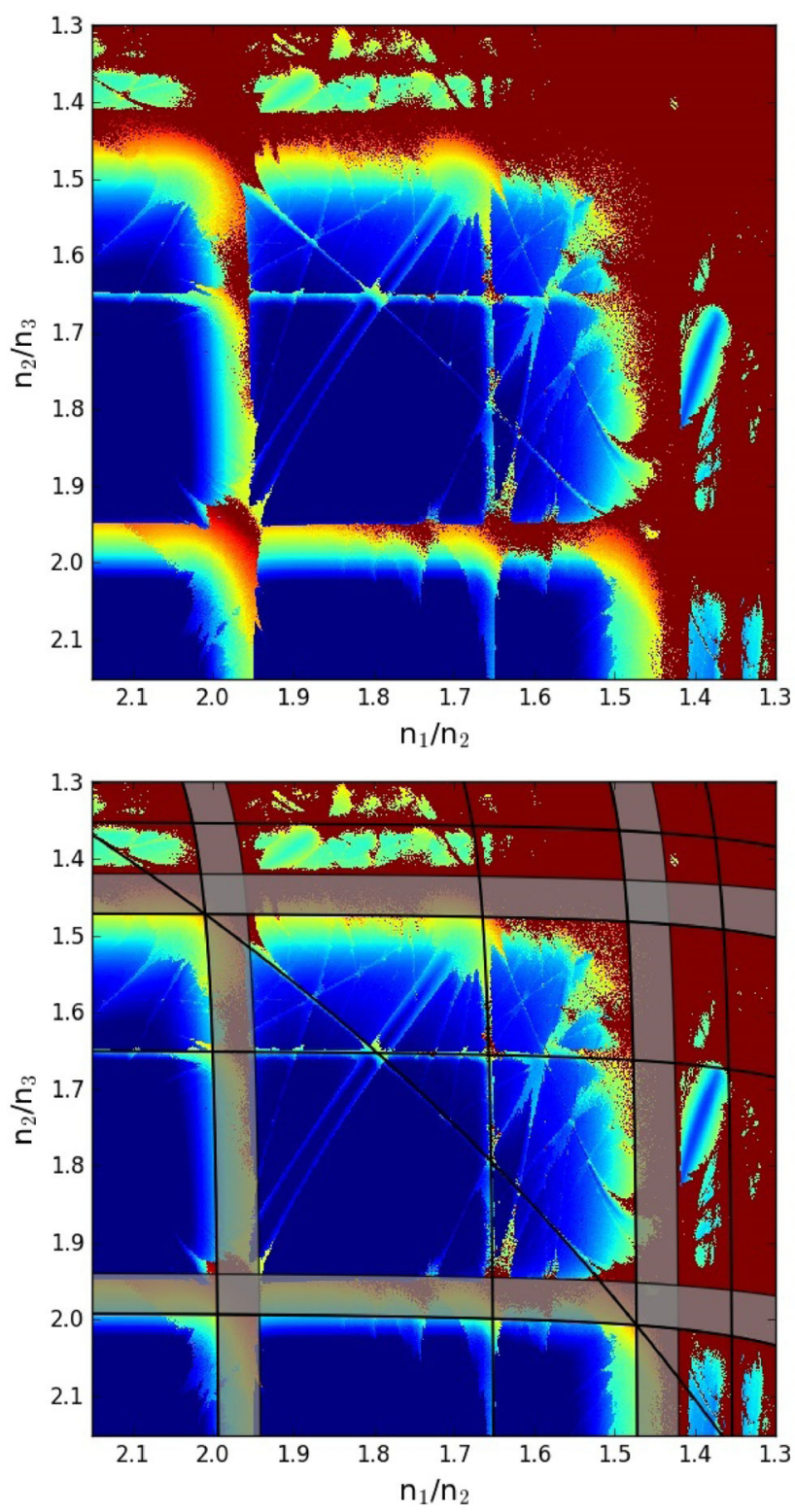

Figure 2. Top: dynamical map of $\max (\Delta a)$ in the $\left(n_{1} / n_{2}, n_{2} / n_{3}\right)$ plane for a grid of $(800 \times 800)$ initial conditions in circular coplanar orbits. Blue tones indicate low changes in semimajor axes, while yellow and red tones indicate increasing values. Total integration times was $T=10^{4}$ orbits of the outer planet. We considered equal-mass planets with $m_{1}=m_{2}=m_{3}=150 m_{\oplus}$ orbiting a central star with $m_{0}=1 \mathrm{~m}_{\odot}$. Bottom: superimposed to the dynamical map, black lines show the nominal location of relevant 2P-MMRs, while for first-order commensurabilities, the distance to the inner separatrix are marked by grey regions. The diagonal curve corresponds to the 3/1 MMR between $m_{1}$ and $m_{3}$.

for some set of $\left(j_{1}, j_{2}, j_{3}\right) \neq(0,0,0)$. Since resonance relations are defined in mean orbital elements, while the dynamical maps are constructed from a grid of initial conditions in osculating elements, we must use the transformation equations deduced in the previous section to relate both sets of variables.

\subsection{Two-planet mean-motion resonances}

Superimposed to the dynamical map, the bottom frame of Fig. 2 shows the main features of two-planet mean-motion resonances (hereafter 2P-MMR). Commensurabilities between $m_{1}$ and $m_{2}$ appear as almost vertical curves, where the curvature is caused by the fact that we are plotting osculating elements and not their mean counterparts. The functional form of the curves were calculated from the expressions deduced in the previous section and are mainly caused by short-period perturbations from the non-resonant third planet (in this case, $m_{3}$ ). From left to right, we observe the $2 / 1,5 / 3$, $3 / 2$, and 7/5 MMRs, whose nominal location is identified by broad black curves. The observed shift with respect to the exact commensurability relations is this time due to the short-period perturbations between both $m_{1}$ and $m_{2}$.

While second-order MMRs have negligible libration widths for circular orbits, first-order resonances cause a significant change in both eccentricity and semimajor axis for all initial conditions between the nominal resonant value and the inner separatrix at $e_{\mathrm{i}}=0$ (see Ramos et al. 2015). This region is shaded in grey, where the semiwidth of the libration region was estimated using the analytical expression by Deck et al. (2013).

The same 2P-MMRs, now between $m_{2}$ and $m_{3}$, are depicted as near-horizontal curves. Once again the broad black curves correspond to the nominal location while, the regions up to the inner separatrix are shown in grey. The structures associated with both two-planet resonances are symmetric with respect to the diagonal line defined by $n_{1} / n_{2}=n_{2} / n_{3}$.

The bottom frame of Fig. 2 also shows evidence of 2P-MMRs between non-adjacent planets. The diagonal curve starting from $\left(n_{1} / n_{2}, n_{2} / n_{3}\right) \simeq(2.15,1.35)$ down to $\left(n_{1} / n_{2}, n_{2} / n_{3}\right) \simeq(1.35$, 2.15) marks the location of the $3 / 1$ resonance between $m_{1}$ and $m_{3}$. Although other similar commensurabilities exist in the plot, they are either weaker (and therefore difficult to visualize) or are located for period ratios closer to unity and drown in the red region of the map. As shown by Delisle (2017), MMRs between non-adjacent planets may play an important role in generating new stable fixed points for three-planet resonances.

\subsection{Three-planet mean-motion resonances}

The planets will lie in the vicinity of a three-planet mean-motion resonance (hereafter 3P-MMR) if their mean motions satisfy the linear equation (14) with $j_{\mathrm{i}} \neq 0 \forall i$. We can rewrite the resonance relation as

$p n_{1}-(p+q-s) n_{2}+q n_{3} \simeq 0$; with $p, s, q \in \mathcal{Z}$.

The sum of the index is equal to $s$, whose absolute value gives the order of the 3P-MMR. Zero-order three-planet commensurabilities, also referred to as Laplace resonances, correspond to $s=0$. All exoplanetary systems currently associated with multiresonant configurations (e.g. GJ876, Kepler-60, Kepler-80, and Kepler-223) lie in Laplace resonances, as are the well known Galilean satellites. So far, only the outer planets of our own Solar system appear to be affected by high-order 3P-MMRs (Murray \& Holman 1999; Guzzo 2005, 2006), possibly leading to chaotic motion in Gigayear time-scales.

The location of 3P-MMRs in the dynamical map define curves given by the functions

$$
\left(\frac{n_{2}}{n_{3}}\right)^{-1}=\frac{(p+q-s)}{q}-\frac{p}{q}\left(\frac{n_{1}}{n_{2}}\right)
$$

As in case of two-planet commensurabilities, these relations are given in mean variables and must be transformed to osculating 

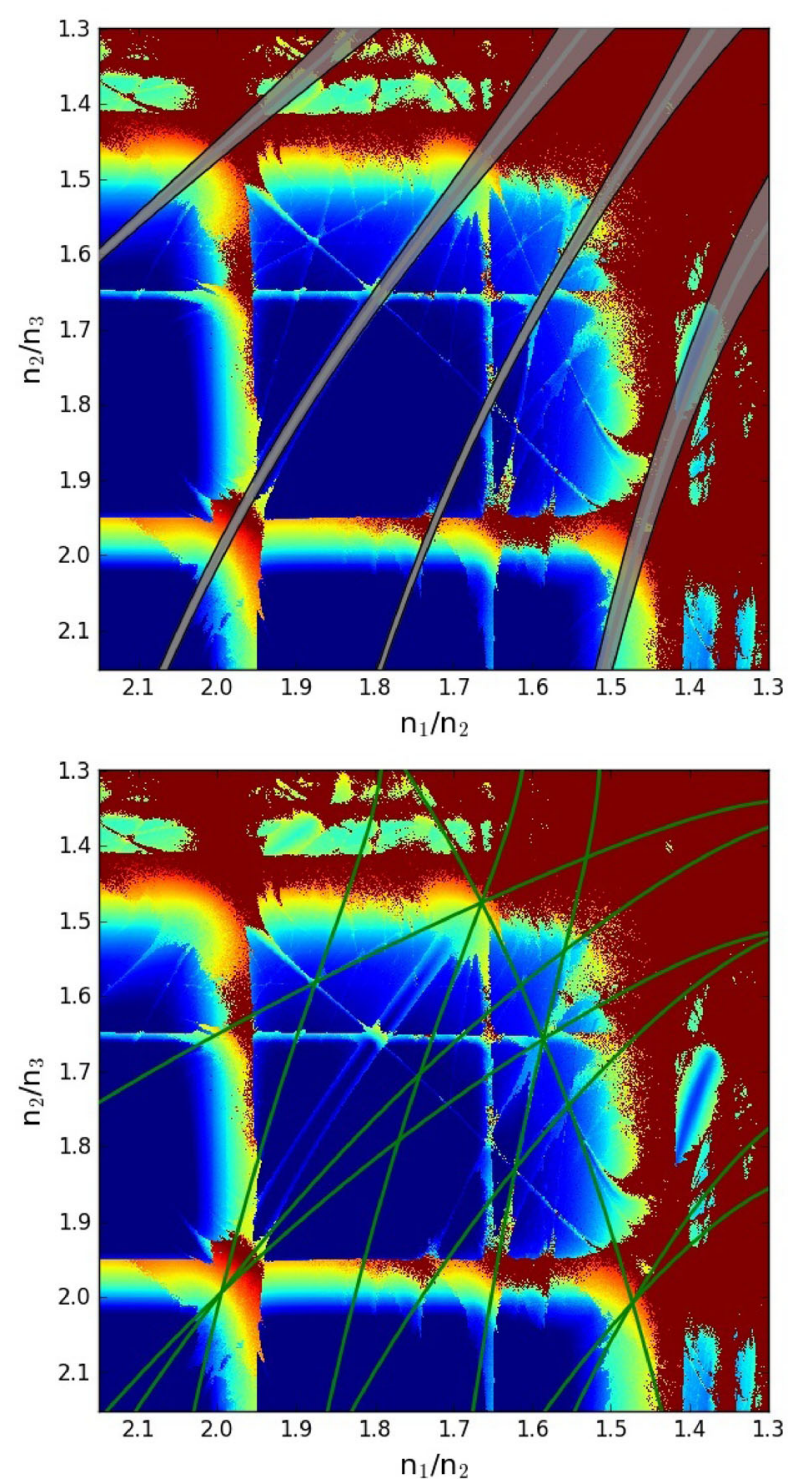

Figure 3. Top: location and libration widths of zero-order (i.e. Laplace) three-planet resonances in the dynamical map described in Fig. 2. From left to right, the commensurabilities correspond to $n_{1}-4 n_{2}+3 n_{3}=0$, $n_{1}-3 n_{2}+2 n_{3}=0,2 n_{1}-5 n_{2}+3 n_{3}=0$, and $n_{1}-2 n_{2}+n_{3}=0$. Bottom: green lines show the position of several first-order three-planet resonances.

elements before plotting them in the representative plane of initial conditions.

The top frame of Fig. 3 shows the location and libration width (for zero eccentricity) of several Laplace resonances $(s=0)$. Although an infinite number of Laplace resonances exist in the plane, we only plotted those MMRs that led to appreciable values of $\max (\Delta a)$ during the integration time-scale. Although this is not a rigorous criterion, these should be the most relevant commensurabilities liable to affect the dynamics of planetary systems, at least for the mass values considered here. The libration widths were calculated with the analytical model by Quillen (2011) and show a very good agreement with the structure of the dynamical map, although the numerical simulations seem to indicate larger libration widths. As shown by Quillen (2011) (see also Gallardo et al. 2016), Laplace resonances have a very week dependence with the eccentricities and both branches of the separatrix are clearly noticeable for circular orbits.

The map also shows evidence of first- and higher order 3PMMRs. The locations of the most relevant first-order commensurabilities are plotted as green curves in the bottom frame of Fig. 3. As with their zero-order cousins, most curves have a positive gradient in the mean-motion-ratio plane (i.e. $\left.\partial\left(n_{2} / n_{3}\right) / \partial\left(n_{1} / n_{2}\right)>0\right)$; the opposite occurs when $q<0$. The only member of this set plotted here corresponds to the $n_{1}+n_{2}-n_{3}=0$ resonance.

We can define two different types of 3P-MMRs. If the subsystems $m_{1}-m_{2}$ and $m_{2}-m_{3}$ are both in two-planet resonances such that $p n_{1}-k_{1} n_{2}=0$ and $k_{2} n_{2}-q n_{3}=0$, then the difference between both will also be zero: $p n_{1}-\left(k_{1}+k_{2}\right) n_{2}+q n_{3}=0$. In this case, the three-planet resonance will only be a consequence of the overlap of two independent 2P-MMRs (Morbidelli 2000) and the dynamics will still be dominated by the individual resonant terms stemming from the first-order normal form, and not by the secondorder perturbation terms modelled by Quillen (2011). We refer to such a configuration as a double resonance. The three outer planets of the Gliese 876 system lie in such a double resonance, where three of the four two-planet critical angles librate leading to a libration of the Laplace angle (Martí, Giuppone \& Beaugé 2013).

The opposite case occurs when the 3P-MMR condition $p n_{1}-(p+q-s) n_{2}+q n_{3}=0$ is satisfied without the individual planetary pairs exhibiting resonant motion. Following Goździewski et al. (2016), we refer to such a configuration as a pure three-planet resonance. Only in these cases are resonant models constructed from the Hamiltonian second-order normal forms valid, since it is expected that the first-order terms should have short periods and near-zero average values. However, as discussed by Gallardo et al. (2016), this is not always the case and the domain of validity of second-order models (e.g. Quillen 2011) may be a strong function of the eccentricities.

Fig. 4 shows additional dynamical maps, this time constructed for lower planetary masses and zooming into mean-motion ratios. For the top and middle graphs, we adopted masses $m_{i}=30 m_{\oplus}$, while in the bottom graph, we used $m_{\mathrm{i}}=3 m_{\oplus}$. While the strongly chaotic and unstable regions are no longer present in these plots, the background value of $\max (\Delta a)$ shows a significant increase as the mean-motion ratio approaches unity. This pronounced colour gradient is caused by the increasing amplitude of short-period variations and complicates the identification of the resonant structures in different regions of the plane. While we could eliminate this effect applying a low-pass digital filter on the output of the numerical integrations, this would have implied an unnecessary increase in the computing time. We then opted for a simpler, and more interesting alternative method.

The middle frame of Fig. 4 repeats the top graph, but where we subtracted the short-period amplitudes

$\Delta a_{\mathrm{i}}=\frac{4 L_{\mathrm{i}}}{\mu_{\mathrm{i}} m_{\mathrm{i}}^{\prime 2}} \Delta L_{\mathrm{i}} ;(i=1,2,3)$,

where $\Delta L_{\mathrm{i}}$ are given by expressions (10). The result effectively reduces the differential background value allowing for a much clearer picture of the structures of the representative plane defining the long-term dynamical evolution. The complex web of resonances are now enhanced and stand out in all the different regions of the mean-motion ratio plane.

The bottom frame shows a similar map, this time drawn for planetary masses $m_{\mathrm{i}}=3 m_{\oplus}$, and again after removing the shortperiod variations. Compared to the intermediate masses (middle plot), as well as to the map discussed in Fig. 2, the change from 

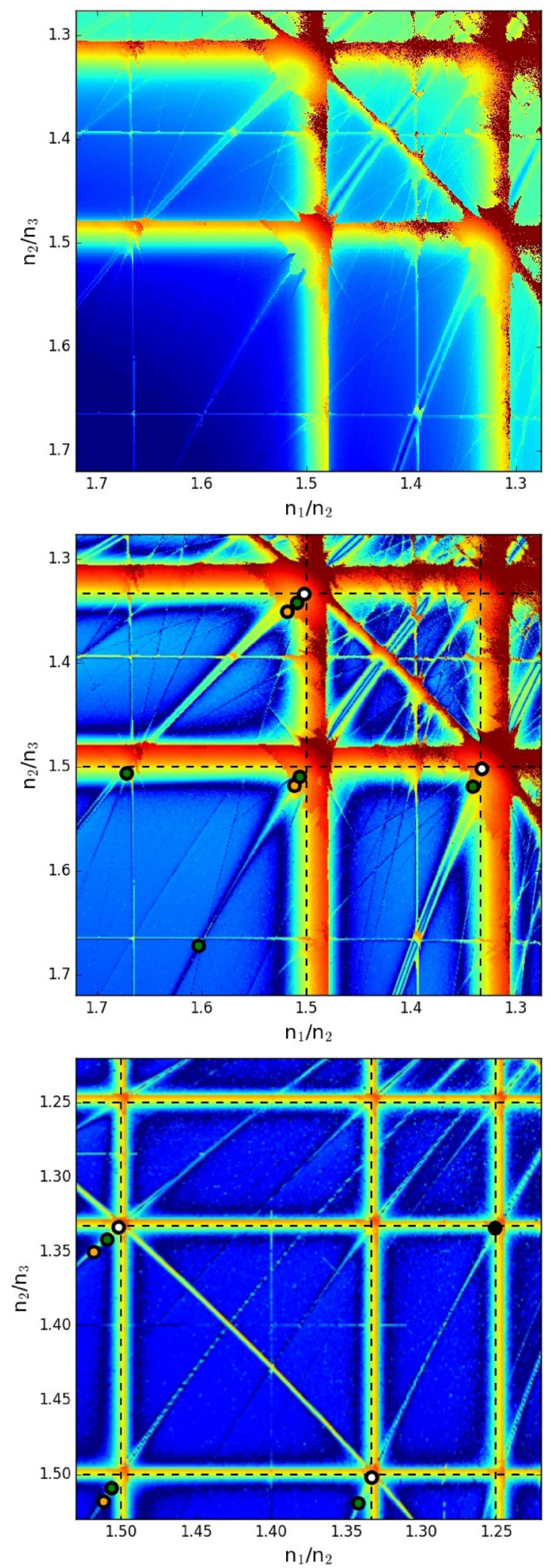

Figure 4. Top: $\max (\Delta a)$ dynamical map for equal-mass planets with $m_{\mathrm{i}}=30 m_{\oplus}$, orbiting a central star with $m_{0}=1 \mathrm{~m} \odot$. Middle: same as above, but after subtracting the amplitudes of the short-period variations. Bottom: dynamical map for $m_{\mathrm{i}}=3 m_{\oplus}$, also without short-period variations. Filled circles in the two lower plots show the location of four exoplanetary systems: Kepler-223 (white), Kepler-60 (black), Kepler-80 (orange), and TRAPPIST-1 (green). Nominal location of first-order 2P-MMRs are identified with horizontal and vertical dashed lines.

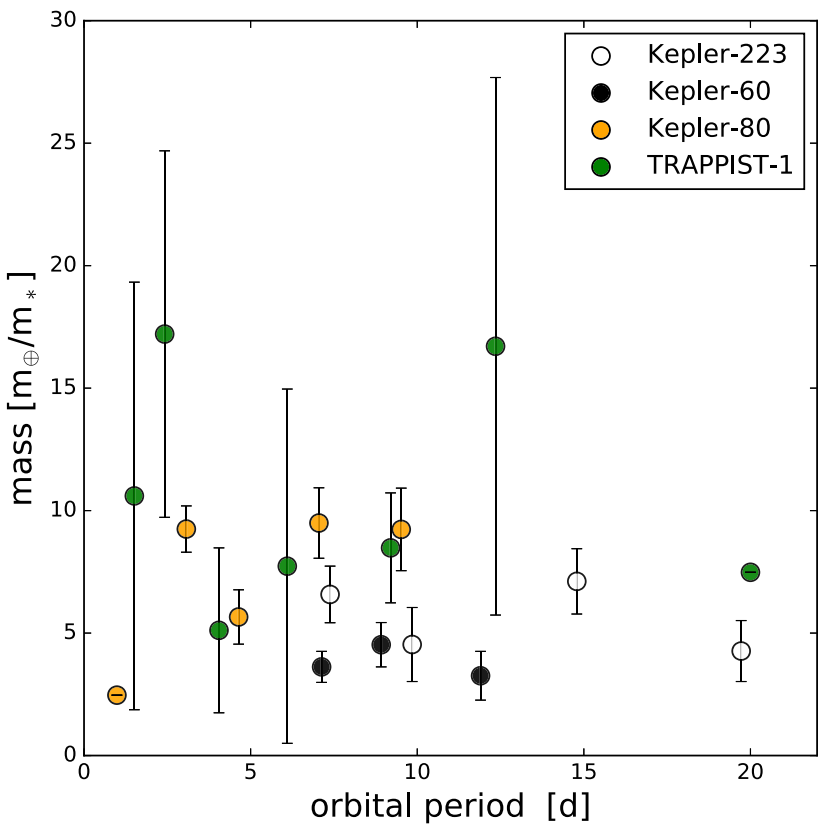

Figure 5. Estimated planetary masses as function of orbital period, for several resonant multiplanetary systems located close to the host star. Colour code is the same as used in the previous figure. Masses are given in units of earth mass divided by stellar mass.

mean to osculating elements is much less pronounced, leading to less deformed structures closer to the nominal value of the meanmotion ratio. The degree of chaoticity (or semimajor axis excitation) is also significantly reduced, although the same is noted for the resonance strengths/widths.

\subsection{Known systems in double resonances}

The two lower plots of Fig. 4 also show the current location of four close-in multiplanet systems whose dynamics is believed to be dominated by three-planet resonances. The colour code employed to identify each system is described in the caption, while the estimated masses and orbital-period distribution are shown in Fig. 5. The masses for both the inner planet of Kepler- 80 and the outer body of TRAPPIST-1 are very uncertain and thus these data have been plotted without error bars. Calculated values of $m_{\mathrm{i}} / m_{*}$ seem to cover the interval between $\sim 3$ and $30 m_{\oplus} / m_{*}$, thus the general qualitative features of their dynamics should correspond to the middle and lower plots of Fig. 4.

While all these systems appear located in double resonances, we can separate them in two distinct groups. The first is comprised of Kepler-60 and Kepler-223, whose location in the mean-motion ratio plane shows no appreciable offset with respect to the nominal location of the double resonances. In the case of Kepler-60, this proximity may be biased since the orbital fit process employed by Goździewski et al. (2016) assumed resonant motion as a proxy. However, this is not the case of Kepler-223, where the libration of the three-planet Laplace angles has recently been measured from TTV data (Mills et al. 2016) and whose proximity to exact resonance appears certain.

TRAPPIST-1 and Kepler-80, representatives of the second group, show a significant displacement with respect to the double resonance, although all the three-planet sub-systems are well aligned with location of the zero-order 3P-MMRs. The orbital-period distribution of these systems (Fig. 5) shows that both are much closer 

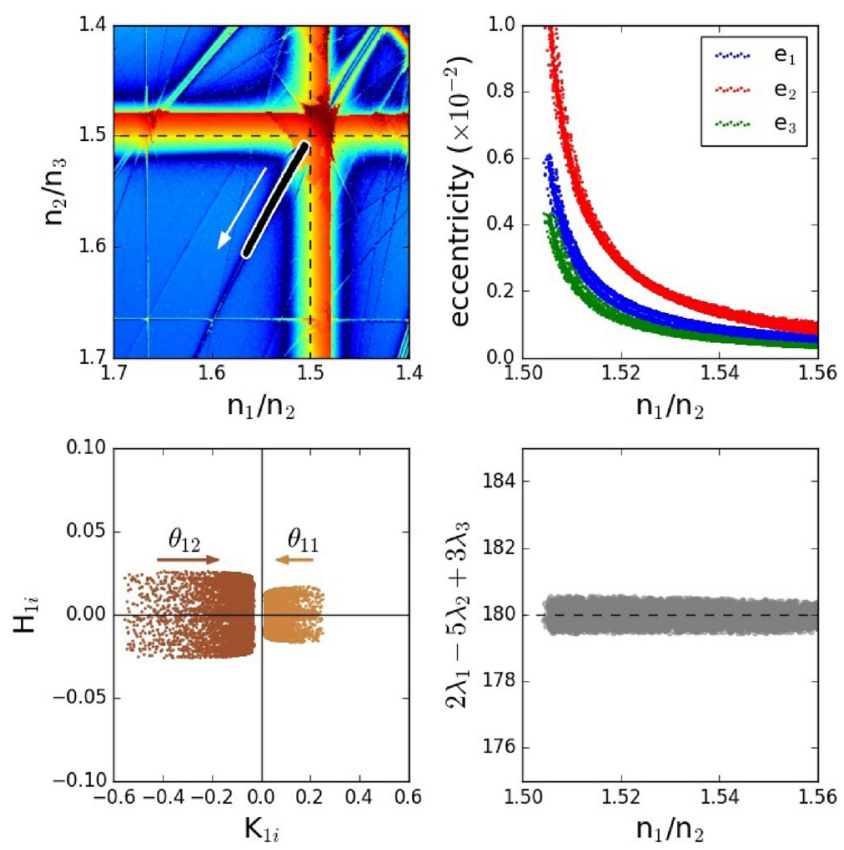

Figure 6. Tidal evolution of a fictitious three-planet system, with masses $m_{1}=7 m_{\oplus}, m_{2}=10 m_{\oplus}$, and $m_{3}=15 m_{\oplus}$, and initially trapped in a $3 / 2$ double resonance. Top left: superimposed to the dynamical map, the blackover-white line shows the evolutionary track of the system, leading towards larger mean-motion ratios, but following the 3P-MMR $2 n_{1}-5 n_{2}+3 n_{3}=0$. Top Right: eccentricities as function of $n_{1} / n_{2}$. Bottom left: behaviour of the regular canonical variables $\left(K_{1 \mathrm{i}}, H_{1 \mathrm{i}}\right)=\left(2 S_{i}\right)^{1 / 2}\left(\cos \theta_{1 \mathrm{i}}, \sin \theta_{1 \mathrm{i}}\right)$, for the resonant angles of the inner pair: $\theta_{1 \mathrm{i}}=3 \lambda_{2}-2 \lambda_{1}-\varpi_{\mathrm{i}}$. Arrows indicate direction of the evolution. Similar behaviour is seen for the pair $\left(K_{2 \mathrm{i}}, H_{2 \mathrm{i}}\right)$. Bottom right: resonant angle of the Laplace resonance as function of $n_{1} / n_{2}$.

to their host stars than the members of the first group, thus more susceptible to tidal evolution. Depending on the number of librating two-planet resonance angles, Batygin \& Morbidelli (2013) and Papaloizou (2015) proposed that some systems within double resonances could evolve by tidal effects preserving the libration of the Laplace angle. Specifically, numerical simulations of Kepler-80 by MacDonald et al. (2016) showed how tidally induced divergent migration may have lead to final orbital architectures similar to the observed system, characterized by large displacements from nominal 2P-MMR while preserving libration of the Laplace angles.

To understand how tides affect the distribution of three-planet resonance chains in the $\left(n_{1} / n_{2}, n_{2} / n_{3}\right)$ plane, Fig. 6 shows the tidal evolution of a fictitious system comprised of three planets orbiting a $m_{*}=1 \mathrm{~m}_{\odot}$ central star. Initial conditions were taken from the final state of a prior simulation of resonance capture, and correspond to a very small amplitude libration of all two-planet resonant angles:

$\theta_{11}=3 \lambda_{2}-2 \lambda_{1}-\varpi_{1} ; \theta_{12}=3 \lambda_{2}-2 \lambda_{1}-\varpi_{2}$

$\theta_{22}=3 \lambda_{3}-2 \lambda_{2}-\varpi_{2} ; \theta_{23}=3 \lambda_{3}-2 \lambda_{2}-\varpi_{3}$.

The tidal evolution was simulated using the classical equilibrium tide model (Mignard 1979) incorporating the precession and dissipation terms into an $N$-body code (e.g. Beaugé \& Nesvorný 2012). Since the graphs present correlations between different projections of the phase space, and not variables as a function of time, the results are independent of the tidal parameters, as long as the evolutionary time-scales are adiabatic with respect to the librational periods.

Starting from $\left(n_{1} / n_{2}, n_{2} / n_{3}\right) \simeq(1.504,1.509)$, the divergent migration increased both mean-motion ratios driving the system away from the double resonance (upper left-hand frame). However, the rates of change are not independent but constrained by the Laplace resonance. As found previously by Papaloizou (2015) and MacDonald et al. (2016), the zero-order 3P-MMR stemming from the double resonance acts as a trench through which the system evolves. As seen in the lower right-hand frame, the corresponding Laplace resonant angle $\phi=2 \lambda_{1}-5 \lambda_{2}+3 \lambda_{3}$ librates around $\phi=180^{\circ}$ with a very small amplitude with no discernible linear deviation.

Although we expected the two-planet resonant angles to circulate once the system increased its offset with respect to the double resonance, the bottom left-hand plot shows this is not the case. The tidal evolution follows the ACR loci of solutions (Beaugé, Michtchenko \& Ferraz-Mello 2006; Michtchenko, Beaugé \& Ferraz-Mello 2006), leading to a monotonic decrease in the eccentricities (upper righthand plot) with damped amplitudes of the secular modes.

While these librations are kinematic and the motion is no longer encompassed by the resonant separatrix, the two-planet resonant terms still seem to be important in defining the dynamics of the system, even far from their nominal locations. This raises the issue of the relative weight between the pure three-planet resonant terms (Quillen 2011) and the two-planet perturbations in defining the longterm dynamics of the system. Perhaps the underestimation of the analytical estimations of the libration width for Laplace resonances (top frame of Fig. 3) is not due to intrinsic limitations in the secondorder normal form, but to the first-order contributions which were not included.

\section{RESONANT CAPTURE IN 3P-MMRS}

The multiresonant extrasolar systems discussed in the previous section are believed to have attained their current configuration as a consequence of a smooth planetary migration with the primordial gaseous disc. Since their masses $m_{\mathrm{i}} / m_{*}$ are small, we expect the orbital decay to have been dominated by a Type-I migration (e.g. Ward 1997).

While in two-planet systems planet-disc interactions drive the mean-motion ratio to a resonance lock in 2P-MMRs, in three-planet cases the differential migration (i.e. mean motion ratio) is only stalled when the complete system is trapped in two independent MMRs. In the examples analysed above, all captures appear to be two-planet resonances. Thus, all two-planet commensurabilities do not appear to be pure but double resonances.

\subsection{The case of TRAPPIST-1}

In this scenario, the current orbital configuration of planets b-c-d of TRAPPIST-1 (see middle plot of Fig. 4) looks curious. According to Gillon et al. (2017), this sub-system is located in a double resonance identified by $\left(n_{1} / n_{2}, n_{2} / n_{3}\right)=(8 / 5,5 / 3)$ and thus corresponding to high-order two-planet resonances. While the dynamical map shows evidence of the $5 / 3$ commensurability, no indication is observed of the third-order 8/5 2P-MMR. However, we do notice a diagonal strip intersecting the observed location of b-c-d corresponding to the first-order 3P-MMR $3 n_{1}-6 n_{2}+2 n_{3}=0$. We then ask what role may three-planet resonances have played in the trapping of these planets and whether the evolutionary tracks of the system may have actually followed 3P-MMRs instead of the traditional two-planet counterparts.

In an attempt to see some light into this issue, we performed a series of $N$-body simulations of type-I planetary migration of TRAPPIST-1-like systems. Instead of introducing an ad hoc exterior force acting only on the outer planet (e.g. Tamayo et al. 

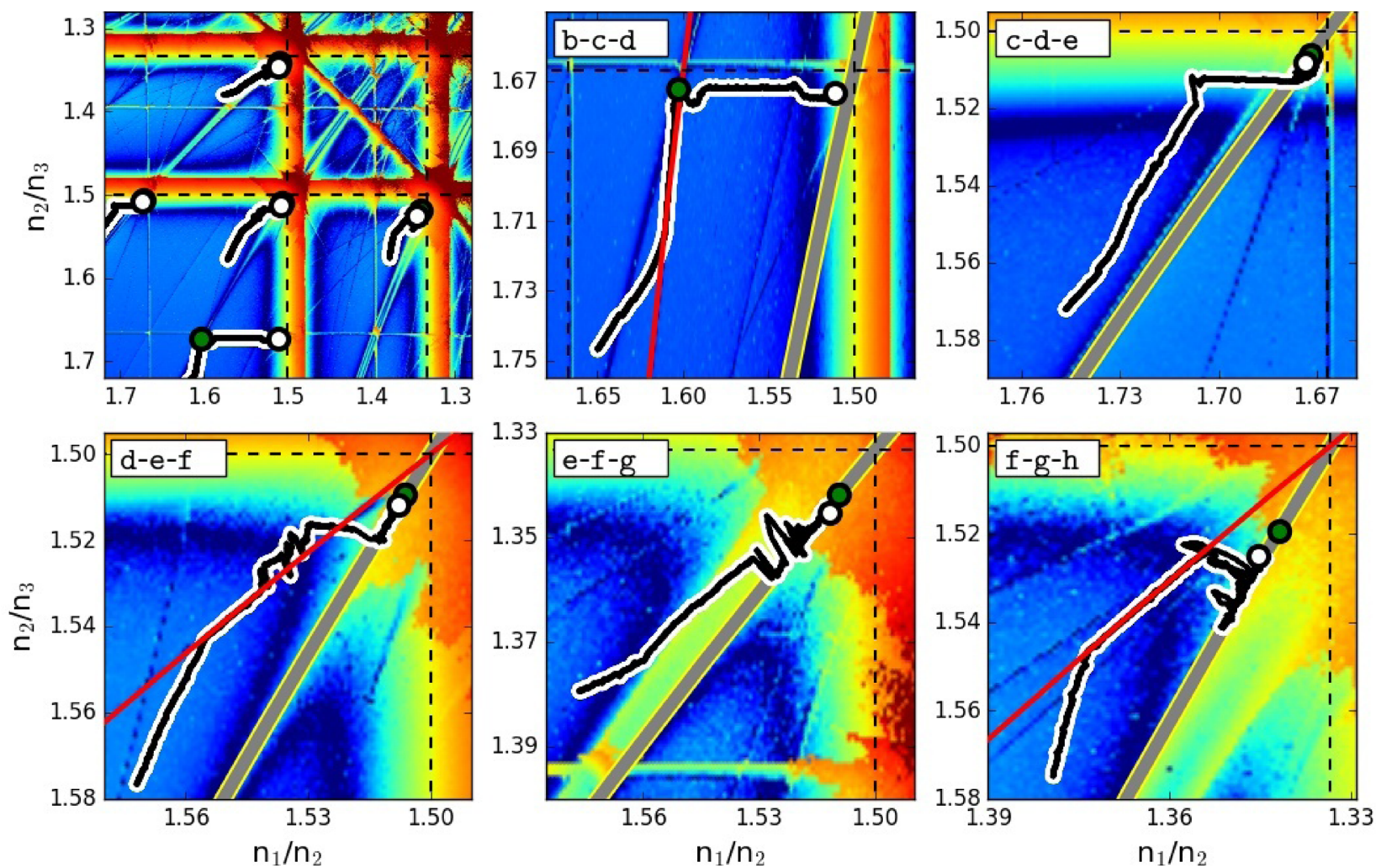

Figure 7. $N$-body simulation of type-I planetary migration in a TRAPPIST-1-like system. Top left-hand plot shows the evolution of the mean-motion ratios of consecutive three-planet sub-systems, with white filled circles showing the final configuration and the observed planets in green. Black-over-white trails are the evolutionary paths followed by each sub-system from the initial conditions to the final equilibrium points. All other frames show detailed zooms in the vicinity of each sub-system, starting from the triplet (b-c-d) (upper middle graph, see inlaid legend) down to (f-g-h) in the lower right-hand frame. Relevant two-planet resonances are identified by dashed black lines, Laplace 3P-MMRs are shown with broad grey curves, while first-order 3P-MMRs are indicated in red. Numerical data were filtered to eliminate short-period and resonant terms in order to reduce spreading, thus the locations of the resonances are drawn in mean (not osculating) variables. To guarantee convergent migration, planetary masses $m_{\mathrm{i}} / m_{*}$ were taken equal to $16,17,17.6,18.4,19.5,21$, and 24 , all in units of $m_{\oplus} / \mathrm{m}_{\odot}$. The numerical integration was stopped when the system achieved a steady-state configuration, corresponding to $\sim 5 \times 10^{5}$ orbital periods of the outer planet at its initial semimajor axis.

2017), we adopted the analytical prescription of Tanaka, Takeuchi \& Ward (2002) and Tanaka \& Ward (2004), incorporating the partial preservation of the angular momentum suggested by Goldreich \& Schlichting (2014). Full equations of motion and further details of the resulting $N$-body code may be found in Ramos et al. (2017) and Zoppetti, Beaugé \& Leiva (2018). Both tidal evolution and relativistic effects were neglected in these simulations.

Since convergent migration required planetary masses increasing with orbital distance, we assumed $m_{\mathrm{b}}=16, m_{\mathrm{c}}=17, m_{\mathrm{d}}=17.6$, $m_{\mathrm{e}}=18.4, m_{\mathrm{f}}=19.5, m_{\mathrm{g}}=21$, and $m_{\mathrm{h}}=24$, all in units of $m_{\oplus} / \mathrm{m}_{\odot}$. Although these values are arbitrary, they are more or less consistent with the estimated masses and uncertainties shown in Fig. 5. We assumed a thin flat laminar disc with $H_{0}=0.05$ and a surface density profile $\Sigma(r)=\Sigma_{0} r^{-\sigma}$ with $\sigma=1 / 2$ and $\Sigma_{0}=50 \mathrm{gr} \mathrm{cm}^{-2}$. This low surface density led to a characteristic migration time-scale of $\tau_{\mathrm{a}} \sim 10^{5} \mathrm{yr}$, probably much higher than expected for an MMSN but practically equal to that assumed by Tamayo et al. (2017).

Initial conditions were chosen with eccentricities $e_{\mathrm{i}}=0.01$ and all angles equal to zero; semimajor axes placed the planets outside (but not very close to) the observed resonance locations. By modifying the planetary masses (i.e mass ratios), we were able to generate evolutionary tracks in the $\left(n_{1} / n_{2}, n_{2} / n_{3}\right)$ plane with any desired angle, and thereby choose which would be the first resonance encountered by each sub-system. This degree of freedom contrasts with the approach adopted by Tamayo et al. (2017) where the sub-systems always started migrating following vertical lines in the mean-motion ratio plane.

Results of a typical run are shown in Fig. 7, superimposed to the dynamical map obtained for $m_{\mathrm{i}}=30 m_{\oplus}$. The top left-hand plot shows a global view, with the evolutionary trails of the migration in black-over-white lines, while the final configuration is highlighted in white filled circles. The current positions of the system is shown in green, although in most cases these practically coincide with the simulated system and are virtually unseen. The only triplet we were not able to reproduce consists of planets (b-c-d) for which our $N$ body integration ultimately led to a capture in $\left(n_{1} / n_{2}, n_{2} / n_{3}\right)=(3 / 2$, $5 / 3)$.

The remaining plots of Fig. 7 focus on the migration of the different sub-system triplets, identified by inlaid legends in the upper left-hand corners. Each will be discussed below.

(i) Planets $(b-c-d)$ : starting from the lower left-hand end of the plot, the three-planet sub-system approaches and is trapped in the $3 n_{1}-6 n_{2}+2 n_{3}=0$ first-order 3P-MMR (red line), thereafter following its trail up to the observed position of the real system and the two-planet resonance $n_{2} / n_{3}=5 / 3$. Notice no indication of the $n_{1} / n_{2}=8 / 5$ in the dynamical map. Although the simulated system is temporarily trapped in a location close to the observed planets, it 
is eventually ejected and follows the $n_{2} / n_{3}=5 / 3$ commensurability until finally resting in $\left(n_{1} / n_{2}, n_{2} / n_{3}\right)=(3 / 2,5 / 3)$. The broad grey line corresponds to the Laplace resonance $4 n_{1}-9 n_{2}+5 n_{3}=0$. Even after several attempts with different masses and disc parameters, we were unable to find any cases of a permanent stable capture in the double resonance $\left(n_{1} / n_{2}, n_{2} / n_{3}\right)=(8 / 5,5 / 3)$. This apparent inconsistency with the results of Tamayo et al. (2017) could be due to differences in the modelling of the planetary migration, or perhaps a more thorough exploration of the parameter space is needed.

(ii) Planets (c-d-e): this is a straightforward case. The direction of relative migration avoids any significant pure 3P-MMR and the outer pair is initially in the $n_{2} / n_{3}=3 / 2$ resonance. Later, migration follows this commensurability until reaching the double resonance $\left(n_{1} / n_{2}, n_{2} / n_{3}\right)=(5 / 3,3 / 2)$ stopping very close to the current location of the observed planet triplet configuration.

(iii) Planets (d-e-f): after an initial migration in a secular configuration, the system is trapped in the first-order $2 n_{1}-7 n_{2}+6 n_{3}=0$ pure 3P-MMR (red line), following its trail until reaching the vicinity of the double resonance where the trajectory begins to exhibit irregular oscillations. At one point, the system leaves the first-order resonance and is trapped in the strong Laplace commensurability defined by $2 n_{1}-5 n_{2}+3 n_{3}=0$ (broad grey line), where it continues to migrate until reaching a final destination very close to the actual planets. The capture into the pure zero-order 3P-MMR does not seem to follow a smooth transition but seems consequence of small-scale scattering caused by perturbations on to the first route followed by the system.

(iv) Planets (e-f-g): contrary to the previous case, this subsystem appears to suffer a smooth capture into the pure Laplace resonance $n_{1}-3 n_{2}+2 n_{3}=0$ (broad grey line) early in its migration, although we cannot rule out a possible first-order 3P-MMR guiding the first part of the integration. However, we were unable to find a commensurability relation of this kind that was sufficiently strong to explain the transition between the initial condition and the Laplace resonance.

(v) Planets ( $\mathrm{f}-\mathrm{g}-\mathrm{h})$ : the final and most interesting example is the sub-system composed of the three outermost planets. At first hand, the overall evolution follows closely that of (d-e-f), with an initial capture in the $3 n_{1}-8 n_{2}+6 n_{3}=0$ resonance (red line) and later switching over to the Laplace $n_{1}-2 n_{2}+n_{3}=0$ 3P-MMR. However, what makes this case particularly noteworthy is the large final offset with respect to the nominal values of the double resonance, even larger than the value measured for the real planets. However, both the simulated and observed planets show no appreciable displacement from the zero-order pure three-planet commensurability.

While we were unable to completely describe the migration and formation of the full resonance chain of the TRAPPIST-1 system, and the present relative location of the three inner planets was not obtained, the results of these simulations have shown unexpected insights into the complex dynamics of multiresonant systems. The first conclusion is that two-planet resonances are not the only commensurabilities capable of trapping multiplanet systems. If the migration time-scale is sufficiently large, first-order pure 3P-MMRs may also lead to capture and guide the system towards additional commensurabilities. Another unexpected result is capture into Laplace-type resonance, although here it is not clear whether these can be reached through a smooth migration or require passage through a chaotic layer generated by the interaction with other resonances. Whatever the explanation, these examples point to a diversity of dynamics much richer than previously imagined.

A second and perhaps more important result is the large resonance offset attained by the bodies without the need of assuming later stage tidal evolution. The sub-system comprised by planets ( $f-g-h)$ is probably the best example where our simulation led to values significantly displaced with respect to the nominal mean-motion ratios, even larger than the observed quantities. Moreover, since this sub-system is the farthest from the central star, it is expected that tidally induced divergent migration would be less important in this case than for the other planet triplets. Perhaps the explanation does not lie in tidal effects, but solely in the resonant dynamics and coupling of the different links involved in the resonant chain.

It is nevertheless necessary to bear in mind that the magnitude of the resonant offset is a strong function of the planetary masses, regardless of whether we assume resonant interactions or tidal effects. For this reason, we do not expect our offsets to be exactly equal to the observed values. However, it is compelling to note that the offsets obtained from our simulation increase for sub-systems farther from the central star, as also appears to be case of the observed TRAPPIST-1 planets.

\subsection{Resonance trapping of fictitious systems}

Given the rich diversity in resonant captures noted in the previous example, we wished to study if other outcomes were also possible. In particular, we wondered whether sufficiently long migration timescales in fictitious three-planet systems could lead to permanent stable captures in resonant configurations that are not associated with double resonances between adjacent planets.

We performed a series of $N$-body simulations similar to that described in the previous sub-section, varying planetary masses, initial semimajor axes, and the surface density of the disc. The corresponding orbital migration time-scales were found to lie in the interval $\tau_{\mathrm{a}} \in\left[10^{4}, 10^{7}\right] \mathrm{yr}$. While fast migrations always led to capture in strong double resonances, slower rates of orbital decay yielded a wider range of possibilities. Finally, to allow for a more direct comparison with compact multiplanet systems, we restricted the masses to $1-30 m_{\oplus}$. We also adopted $m_{0}=1 \mathrm{~m}_{\odot}$ for simplicity.

Fig. 8 shows the results of three simulations showing diverse outcomes. The top two frames correspond to an $N$-body run with planetary masses $m_{1}=16, m_{2}=19$, and $m_{3}=22$, all in units of Earth masses. For the disc surface density profile $\sigma=1 / 2$, these mass ratios guaranteed convergent migration, seen in the dynamical map as an initial diagonal evolutionary track leading towards $n_{\mathrm{i}} / n_{\mathrm{i}+1} \rightarrow 1$. The surface density of the disc at $r=1$ au was chosen equal to $\Sigma_{0}=20 \mathrm{gr} \mathrm{cm}^{-} 2$.

First the two inner planets are trapped in the 5/3 2P-MMR, after which the system continues to evolve vertically until reaching the $n_{1}-3 n_{3}=0$ commensurability. This corresponds to a 3/1 2P-MMR between the inner and outer planets and may be seen in the map as a diagonal line crossing the graph in an obtuse angle. Although planetary migration does not stop and all semimajor axes continue to decrease, the system arrived at a stable stationary solution with eccentricities of the order of $e_{\mathrm{i}} \sim 10^{-3}$ and no further secular change in the mean-motion ratios. The right-hand plot shows the temporal behaviour of the resonant angles $5 \lambda_{2}-3 \lambda_{1}-2 \varpi_{2}$ (grey) and $3 \lambda_{3}-\lambda_{1}-2 \varpi_{1}$ (blue). Both librate around symmetric values indicating that the system is in fact trapped in an orbital configuration in which the inner planet is simultaneously in a two-planet MMR with the middle and outer planets (respectively), but $m_{2}$ and $m_{3}$ are not themselves in a resonant motion. 

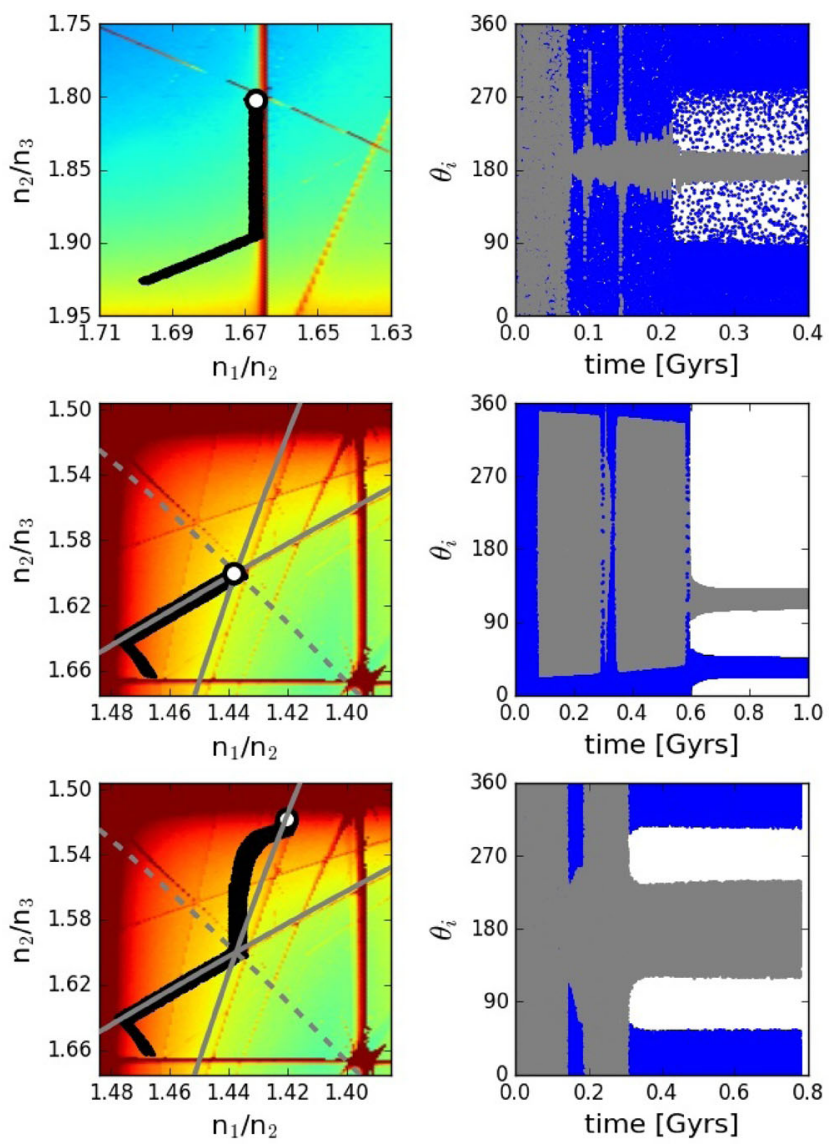

Figure 8. Results of three $N$-body simulations of fictitious three-planet systems. Black dots in the left-hand plots show the evolutionary tracks in the $\left(n_{1} / n_{2}, n_{2} / n_{3}\right)$ superposed over the dynamical map constructed for $m_{\mathrm{i}}=30 m_{\oplus}$. Grey continuous lines show the location of the $2 n_{1}-6 n_{2}+5 n_{3}=0$ and $4 n_{1}-7 n_{2}+2 n_{3}=0$ first-order 3P-MMRs, while dashed lines correspond to the second-order $2 n_{1}-n_{2}-3 n_{3}=0$ resonance. The locations of these resonances have been plotted in mean elements and thus show a displacement with respect to the dynamical map. Right-hand plots show the temporal behaviour of resonant angles involved in each simulation. See the text for details.

The two middle frames (left and right) show the result of a second simulation. This time we adopted masses $m_{1}=18.4, m_{2}=19$, and $m_{3}=22$ (in units of $m_{\oplus}$ ), which implies a slight increase in the inner mass with respect to the previous case. The aim was to generate an initial divergent migration between $m_{1}$ and $m_{2}$ and analyse how the full system reacted to this non-trivial situation. The surface density of the disc was left unchanged, but the initial separations between the planets was reduced in order to study a region of the phase space more densely populated by three-planet resonances.

As before, the evolutionary track in the plane of mean-motion ratios is depicted in the left-hand plot. The initial divergence of the inner planetary pair is stopped as soon as the system encounters and is trapped in the $2 n_{1}-6 n_{2}+5 n_{3}=0$ first-order $3 \mathrm{P}$ MMR. From this point onwards, the corresponding resonant angle $2 \lambda_{1}-6 \lambda_{2}+5 \lambda_{3}-\varpi_{3}$ begins to librate around an asymmetric centre (blue dots in right-hand graph), although it suffers a temporary circulation as it suffers a tangential pass through another commensurability during its path. The subsequent migration follows the $2 n_{1}-6 n_{2}+5 n_{3}=0$ family until it encounters the $4 n_{1}-7 n_{2}+2 n_{3}=0$ resonance. This intersection of two independent 3P-MMRs acts as a planetary trap, effectively stalling any

additional differential migration. From this point onwards the critical angle $4 \lambda_{1}-7 \lambda_{2}+2 \lambda_{3}+\varpi_{3}$ also begins to exhibit a libration, also around an asymmetric solution, while the eccentricities remain only marginally excited at $e_{\mathrm{i}} \sim 10^{-3}$.

The permanent and dynamically stable capture into two independent first-order 3P-MMR is a previously unknown outcome of slow migrations in three-planet systems. The second-order three-planet resonance marked with dashed lines in the dynamical map did not show any appreciable dynamical effects in the system. However, it is interesting to note that

$\left(4 n_{1}-7 n_{2}+2 n_{3}\right)-2\left(2 n_{1}-6 n_{2}+5 n_{3}\right)=5 n_{2}-8 n_{3}$

which implies that a simultaneous libration in both first-order 3PMMRs will also lead to a libration of the two outer planets in the $n_{2} / n_{3}=8 / 5$ resonance. This is the same commensurability which is believed to dominate planets (b-c-d) of TRAPPIST- 1 .

The question now is to elucidate which resonances are the cause and which is the consequence. At first hand, we would expect that even a third-order two-planet resonance such as the $8 / 5$ commensurability would be more significant than a first-order 3P-MMR. However, the absence of any indication of the $8 / 5$ resonance in the dynamical map raises some doubts.

Although the intersection of two independent first-order 3PMMR is always associated with 2P-MMRs between adjacent planets, many times these are of high order and thus dynamically negligible. For example, while the interaction of the three-planet commensurabilities discussed in the middle plot of Fig. 8 lead to a 8/5 resonance between $m_{2}$ and $m_{3}$, the corresponding mean-motion ratio of the inner planetary pair is $n_{1} / n_{2}=23 / 16$, a very high-order commensurability of dubious influence. Consequently, it is possible that the capture process of both TRAPPIST-1 and the fictitious system in Fig. 8 may actually be dominated by first-order 3P-MMRs and not by high-order 2P-MMRs.

The two bottom frames of Fig. 8 correspond to a third simulation, with exactly the same masses and initial conditions as before, but with a higher disc surface density: $\Sigma_{0}=40 \mathrm{gr} \mathrm{cm}^{-2}$. Although the first stages of the migration process are similar, the faster migration can no longer be counterbalanced by the intersection of both first-order 3P-MMRs. After a temporary capture, the system passes through and continues to evolve towards a more compact configuration. However, after a certain time, the planets again encounter the $4 n_{1}-7 n_{2}+2 n_{3}=0$ resonance and the capture is repeated, as seen by the behaviour of the critical angle $4 \lambda_{1}-7 \lambda_{2}+2 \lambda_{3}+\varpi_{3}$ (grey dots in the right-hand plot).

The final lap of the evolution follows this resonant family until it encounters the two planets $n_{2} / n_{3}=3 / 2$ and all further differential migration stops. The blue dots in the right-hand plot show the behaviour of $3 \lambda_{3}-2 \lambda_{2}-\varpi_{2}$, indicating a moderate-amplitude libration around zero and a stable orbital configuration. This simulation therefore shows a different possible outcome of the migration of three-planet systems, in which the pair of resonances acting as a planetary trap is composed of a first-order 3P-MMR plus a (more classical) first-order two-planet commensurability.

The three $N$-body simulations shown in Fig. 8 show completely different outcomes. While in all cases, the relative migration is only halted at the intersection of two independent resonances, these are not restricted to $2 \mathrm{P}-\mathrm{MMRs}$ but may include a wide range of possibilities. Interestingly, none of these final configurations would be identified as three-planet resonances just from the individual (two planet) mean-motion ratios, but only after a detailed analysis of the complete three-planet system. The dynamical maps and the 


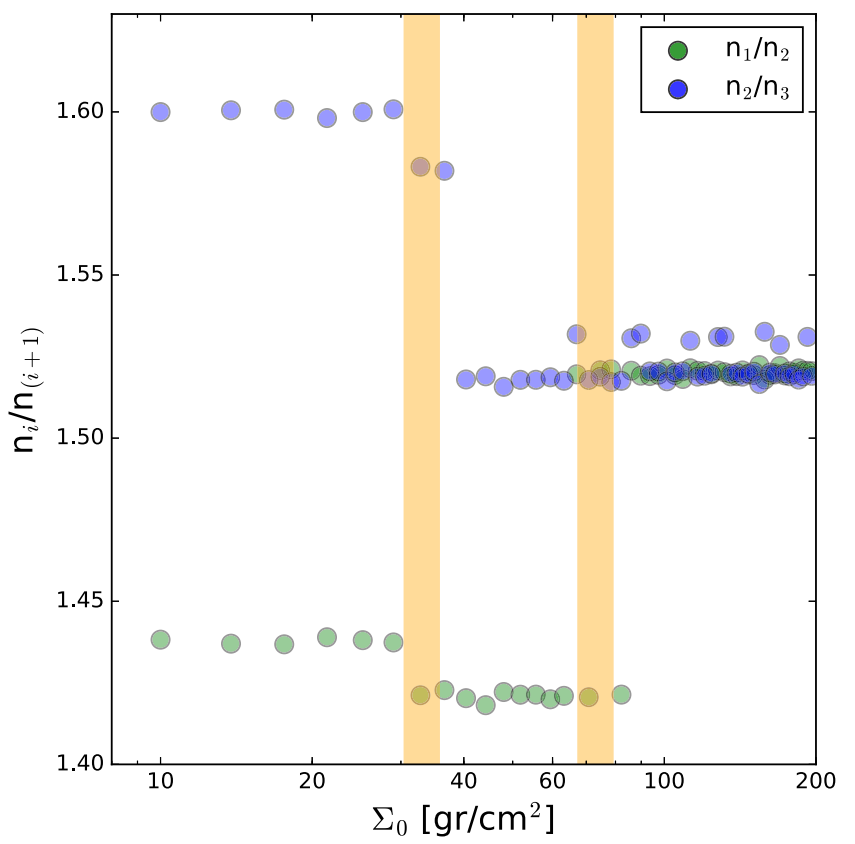

Figure 9. Final values of the mean-motion ratios of a series of $N$-body simulations of migration of three-planet systems with different disc surface densities $\Sigma_{0}=\Sigma(r=1)$. Planetary masses and initial conditions were taken equal to those discussed in the middle and lower frames of Fig. 8. Broad orange vertical lines indicate rough limits for three distinct outcomes. For $\Sigma_{0} \lesssim 30 \mathrm{gr} \mathrm{cm}^{-2}$, all captures occur in the intersection of two first-order 3P-MMRs, while for $\Sigma_{0} \gtrsim 80 \mathrm{gr} \mathrm{cm}^{-2}$ the planets are halted in a $3 / 2$ double resonance. For intermediate surface densities, the systems evolve towards an intersection between a two-planet and a 3-planet commensurability.

identification of relevant multiplanet resonances prove important tools to aid in such a search.

Finally, in order to analyse how the final orbital configuration depends on the migration time-scale, we repeated the previous simulation for a total of 50 values of the disc surface density in the interval $\Sigma_{0} \in[10,200] \mathrm{gr} \mathrm{cm}^{-} 2$. For each run, we calculated the final equilibrium values of $n_{1} / n_{2}$ and $n_{2} / n_{3}$, plotting their values as function of $\Sigma_{0}$. Results are shown in Fig. 9.

For surface densities $\Sigma_{0} \lesssim 30 \mathrm{gr} \mathrm{cm}^{-2}$, corresponding to migration characteristic time-scales $\tau_{\mathrm{a}} \gtrsim 1.5 \times 10^{5} \mathrm{yr}$, the system is captured in an orbital configuration analogous to that described in the middle plots of Fig. 8. In other words, the relative orbital decay is stalled by the apparent intersection of two independent first-order 3P-MMRs. Since a linear combination of both resonant relations yields $5 n_{2}-8 n_{3}=0$, the two outer planets are also seen to be affected by this high-order two-planet commensurability.

For slightly larger surface densities, leading to $\tau_{\mathrm{a}}$ roughly between $8 \times 10^{4}$ and $1.5 \times 10^{5} \mathrm{yr}$, the combined effects of both the $2 n_{1}-6 n_{2}+5 n_{3}=0$ and $4 n_{1}-7 n_{2}+2 n_{3}=0$ resonances are not strong enough to act as a planetary trap and the system evolves towards a new stationary solution involving the $4 n_{1}-7 n_{2}+2 n_{3}=0$ three-planet resonance and the $2 n_{2}-3 n_{3}=0$ two-planet commensurability. However, this orbital configuration only appears possible for a limited range of disc densities and constitute a transition between the low- and high-density scenarios.

Last of all, for planetary migrations corresponding to $\tau_{\mathrm{a}} \lesssim 8 \times 10^{4}$ yr, no 3P-MMR appears sufficiently strong to counteract the dissipative effects and the planets are finally captured in a $3 / 2$ double resonance. All these outcomes were found to be dynamically stable and at least one of the resonant angles was observed to librate around a stationary point with low-to-moderate amplitudes.

Of course, the limit between these different regimes depends on the masses of the planets, as well as other disc parameters such as the flare index and surface density slope. More complex physics (e.g. radiative discs or localized dead zones) may also affect these numerical values and alter the effective reach of the three-planet resonances.

\section{CONCLUSIONS}

Recent discoveries of compact multiplanet systems have revealed several cases of resonant chains (e.g. Kepler-60, Kepler-80, Kepler223, and TRAPPIST-1) comprised of interlocked two- and threeplanet commensurabilities. Although these systems are believed to display complex dynamical behaviour, including the possibility of numerous independent asymmetric librational solutions (Delisle 2017), all three-planet commensurabilities have so far been associated with double resonances and not to pure 3P-MMRs.

In this paper, we have unveiled a more general view of the gravitational interaction of three-planet systems, including a global catalogue of MMRs and possible evolutionary routes from secular to resonant configurations. Our study is based on the construction and detailed analysis of dynamical maps in the mean-motion ratio representative plane of initial conditions. These maps uncovered an extremely rich diversity of possible resonant configurations, including zero- (Laplace-type) and first-order pure 3P-MMRs. Although resonances are dense in the representative plane, not all are equally important. In the absence of adequate analytical models, these maps allowed us to evaluate their relative strengths and identify which could be relevant to the orbital evolution of three-planet systems.

While commensurability relations are defined in mean variables, the representative planes of initial conditions were chosen in osculating elements. While the difference between both sets is usually neglected, in our case, it proved important generating a significant shift in the position of the resonances with respect to the nominal values. To solve this problem, we constructed and applied a simple analytical model for the transformation between mean and osculating semimajor axes. This model proved vital to properly identify which 3P-MMRs were associated with each dynamical feature of the map. As an added bonus, this analytical model allowed us to eliminate the background orbital excitations generated by shortperiod perturbations, thus enhancing the long-term dynamical effects throughout the different regions of the representative plane.

It is important to stress that the maps were drawn for equal-mass bodies for only three specific values of the planetary masses, and thus they are not expected to be exactly the same for any other set $\left(m_{1}, m_{2}, m_{3}\right)$. Nevertheless, their general features and resonance locations should still be qualitatively correct, at least for masses in the same overall range. Thus, even if only illustrative, we have extensively used these generic maps as benchmarks in which to analyse the dynamical interactions of real and fictitious planetary systems.

The effective strength of first-order pure 3P-MMRs was tested with a series of $\mathrm{N}$-body simulations of type-I migration. For fictitious three-planet systems, we found that a complete resonance chain may be formed even if the differential migration between some pairs was initially divergent. Relative migration was only stalled once the system was trapped in two independent MMRs. For short migration time-scales, the intervening commensurabilities are 2P-MMRs, such as those associated with Kepler-60, Kepler-80, and Kepler-223. However, we also found that slower migration rates lead 
to a wider range of possibilities, and multiplanet systems may be trapped in a combination of two- and pure first-order three-planet resonances. Depending on the masses, there always seems to exist an upper limit for the disc surface density, under which two independent pure first-order 3P-MMRs may effectively trap the system into a permanent and stable configuration not associated with any 2P-MMR.

The possibility of resonant chains not involving two-planet resonances is intriguing, since such a system would not be easily identified as multiresonant just by plotting the mean-motion ratio of adjacent planets. Multiplanet captures such as that depicted in the middle frames of Fig. 8 would also not be associated with a three-planet resonance. This raises the question if the distribution of known multiplanet systems may indeed harbour examples of such configurations. We are currently analysing this possibility, and although no global correlation has been found, some individual systems seem promising.

We applied our dynamical maps and migration simulations to the case of TRAPPIST-1 system. Starting from initial separations close to but wider than the current system, we found that most planet triplets halt their relative migration in the double resonance observed today. However, the evolutionary routed towards these nesting places were usually guided by first-order 3P-MMRs and that some captures into Laplace-type 3P-MMRs were also possible before the two-planet commensurabilities are attained.

A curious case is that of the inner planets $(\mathrm{b}-\mathrm{c}-\mathrm{d})$ of TRAPPIST1 , believed to lie in the $n_{1} / n_{2}=8 / 5$ and $n_{2} / n_{3}=5 / 3$ double resonance. We could not find initial conditions or disc parameters leading to a stable capture into this orbital configuration, although some temporary librations were detected for some parameters. However, this difference in results with respect to Tamayo et al. (2017) could be due to differences in the migration prescription or the adopted disc parameters. Although our dynamical maps were able to detect signatures from a wide range of different resonances, we found no evidence of the 8/5 two-planet commensurability in either $\max (\Delta a)$ nor $\max (\Delta e)$. Since this is a third-order resonance, its absence could be due to initial circular orbits. However, it could also point to a case similar to that shown in the bottom plots of Fig. 8 in which the simultaneous libration of a $2 \mathrm{P}-\mathrm{MMR}$ and a first-order pure 3P-MMR combine to show a libration in the 8/5 two-planet resonance even if this commensurability was not an active ingredient in the capture process. As we showed in Fig. 8, such a final configuration is possible only for a limited range of migration timescales, which could also explain why we were not able to reproduce it in our applications to TRAPPIST-1.

Finally, notwithstanding planets $(\mathrm{b}-\mathrm{c}-\mathrm{d})$, our tidal-free capture simulations of TRAPPIST-1 led to two-planet resonance offsets similar to those currently observed for the real planets. It then appears possible that tidal evolution in multiresonance systems may not have played such an important role as previously believed. However, similar studies in other systems (e.g. Kepler-80) are necessary before proposing that these findings are general and not restricted to this particular case.

\section{ACKNOWLEDGEMENTS}

The authors would like to express their gratitude to the computing facilities of IATE and UNC, without which these numerical experiments would not have been possible. We also thank an anonymous referee for valuable comments and suggestions that improved the manuscript. This work was funded by research grants from SECYT/UNC, CONICET, and ANPCyT.

\section{REFERENCES}

Batygin K., Morbidelli A., 2013 AJ, 145, 1

Beaugé C., Nesvorný D., 2012, ApJ, 751, 119

Beaugé C., Michtchenko T. A., Ferraz-Mello S., 2006, MNRAS, 365, 1160

Brouwer D., Clemence G. M., 1961, Methods of Celestial Mechanics. Academic Press, New York

Campante T. L. et al., 2015, ApJ, 799, 170

Deck K. M., Payne M., Holman M. J., 2013, ApJ, 774, 129

Delisle J.-B., 2017, A\&A, 605, A96

Dvorak R., Pilat-Lohinger E., Schwarz R., Freistetter F., 2004, A\&A, 426, L37

Ferraz-Mello S., 2007, Canonical Perturbation Theories - Degenerate Systems and Resonance. Springer, Berlin

Ferraz-Mello S., Michtchenko T. A., Beaugé C., 2005, ApJ, 621, 473

Gallardo T., Coito L., Badano L., 2016, Icarus, 274, 83

Gillon M. et al., 2016, Nature, 533, 221

Gillon M. et al., 2017, Nature, 542, 456

Goldreich P., Schlichting H. E., 2014, AJ, 147, 32

Goździewski K., Migaszewski C., Panichi F., Szuszkiewicz E., 2016, MNRAS, 455, L104

Guzzo M., 2005, Icarus, 174, 273

Guzzo M., 2006, Icarus, 181, 475

Hori G.-I., 1961, AJ, 66, 286

Libert A.-S., Henrard J., 2007, A\&A, 461, 759

Luger R. et al., 2017, Nat. Astron., 1, 0129

MacDonald M. G. et al., 2016, AJ, 152, 105

Martí J. G., Giuppone C. A., Beaugé C., 2013, MNRAS, 433, 928

Michtchenko T. A., Beaugé C., Ferraz-Mello S., 2006, Celest. Mech. Dyn. Astron., 94, 411

Mignard F., 1979, Moon Planets, 20, 301

Mills S. M., Fabrycky D. C., Migaszewski C., Ford E. B., Petigura E., Isaacson H., 2016, Nature, 533, 509

Morbidelli A., 2000, Modern Celestial Mechanics. Cambridge Univ. Press, Cambridge

Murray N., Holman M., 1999, Science, 283, 1877

Papaloizou J. C. B., 2015, Int. J. Astrobiol., 14, 291

Quillen A. C., 2011, MNRAS, 418, 1043

Ramos X. S., Correa-Otto J. A., Beaugé C., 2015, Celest. Mech. Dyn. Astron., 123, 453

Ramos X. S., Charalambous C., Benítez-Llambay P., Beaugé C., 2017, A\&A, 602, A101

Steffen J. H. et al., 2013, MNRAS, 428, 1077

Tamayo D., Rein H., Petrovich C., Murray N., 2017, ApJ, 840, L19

Tanaka H., Ward W. R., 2004, ApJ, 602, 388

Tanaka H., Takeuchi T., Ward W. R., 2002, ApJ, 565, 1257

Tisserand F., 1889, Bull. Astron. Ser. I, 6, 289

Ward W. R., 1997, Icarus, 126, 261

Wisdom J., 1980, AJ, 85, 1122

Yoder C. F., 1979, Nature, 279, 767

Zoppetti F., Beaugé C., Leiva A.-M., 2018, MNRAS, submitted

This paper has been typeset from a $\mathrm{T}_{\mathrm{E}} \mathrm{X} / \mathrm{LT} \mathrm{E} \mathrm{X}$ file prepared by the author. 